\title{
Infectious Otitis Externa
}

National Cancer Institute

\section{Source}

National Cancer Institute. Infectious Otitis Externa. NCI Thesaurus. Code C3299.

Inflammation of the anatomical structures of the outer ear and ear canal secondary to

an infectious process. Bacterial etiology is most common, but fung al infection is also possible. Symptoms include erythema, edema, and pain. 